\title{
SOME FIXED POINT THEOREMS FOR COMPACT MAPS AND FLOWS IN BANACH SPACES $\left({ }^{1}\right)$
}

\author{
BY
}

W. A. HORN

\begin{abstract}
Let $S_{0} \subset S_{1} \subset S_{2}$ be convex subsets of the Banach space $X$, with $S_{0}$ and $S_{2}$ closed and $S_{1}$ open in $S_{2}$. If $f$ is a compact mapping of $S_{2}$ into $X$ such that $\bigcup_{j=1}^{m} f^{\prime}\left(S_{1}\right) \subset S_{2}$ and $f^{m}\left(S_{1}\right) \cup f^{m+1}\left(S_{1}\right) \subset S_{0}$ for some $m>0$, then $f$ has a fixed point in $S_{0}$. (This extends a result of F. E. Browder published in 1959.) Also, if $\left\{T_{t}: t \in R^{+}\right\}$ is a continuous flow on the Banach space $X, S_{0} \subset S_{1} \subset S_{2}$ are convex subsets of $X$ with $S_{0}$ and $S_{2}$ compact and $S_{1}$ open in $S_{2}$, and $T_{t_{0}}\left(S_{1}\right) \subset S_{0}$ for some $t_{0}>0$, where $T_{t}\left(S_{1}\right)$ $\subset S_{2}$ for all $t \leqq t_{0}$, then there exists $x_{0} \in S_{0}$ such that $T_{t}\left(x_{0}\right)=x_{0}$ for all $t \geqq 0$. Minor extensions of Browder's work on "nonejective" and "nonrepulsive" fixed points are also given, with similar results for flows.
\end{abstract}

Interest in fixed point theorems in Banach and locally convex topological linear spaces has been increasing during the past few years as more applications are found in the field of differential and integral equations ([4], [7], [9], [10], [11], and [12]). An important class of fixed point theorems deals with compact or completely continuous mappings on convex sets, going back to the Schauder [13] and Tychonoff [14] theorems, wherein a convex set is assumed mapped into itself, and continuing to more complicated recent theorems such as those of F. E. Browder ([1], [2], and [3]) and others.

The theorems of [1], [2], and [3] deal with the action of iterates of a given mapping, rather than the mapping itself, and it is the purpose of this paper to extend these theorems in the same vein and to prove analogous theorems related to flows, i.e., semigroups of mappings indexed by the nonnegative real half line. This latter extension was suggested to the author by A. J. Goldman of the National Bureau of Standards.

A. Extensions of Browder's first result. This section will generalize the work of Browder in [1]. The result to be generalized is Theorem 2 of [1].

Let $S$ and $S_{1}$ be open convex subsets of the Banach space $X, S_{0}$ a closed convex subset of $X, S_{0} \subset S_{1} \subset S, f$ a compact mapping of $S$ into $X$. Suppose that, for a

Received by the editors January $9,1968$.

AMS Subject Classifications. Primary 4610, 5485.

Key Words and Phrases. Banach space, fixed points, asymptotic fixed point theorems, compact mappings, flows, nonejective fixed points, nonrepulsive fixed points.

(1) A portion of this paper was contained in a dissertation written under the direction of G. Stephen Jones and presented to the University of Maryland in partial fulfillment of the requirements for the Ph.D. degree. 
positive integer $m, f^{m}$ is well defined on $S_{1}, \bigcup_{j=0}^{m-1} f^{j}\left(S_{0}\right) \subset S_{1}$, while $f^{m}\left(S_{1}\right) \subset S_{0}$. Then $f$ has a fixed point in $S_{0}$.

The first lemma is a restatement of Lemma 1 of [1].

Lemma 1. Let $A$ be a simplicial complex and suppose that $f$ is a self-mapping of $A$ such that for some integer $m>0, f^{m}(A)$ is contained in a closed acyclic subset $B$ of $A$. Then $f$ has a fixed point.

Lemma 2 gives the basic innovation on the work of [1]. A variant of this lemma was originally published in [8] and another variant was given by Browder in [2].

LEMMA 2. Given a complex $K$, let $K_{0}$ be a subcomplex and $C_{0}$ a closed, bounded, acyclic subset of $K_{0}$. Suppose that $f$ is a simplicial mapping of the nth barycentric subdivision of $K$ into $K$ such that, for some positive integer $m, f^{j}\left(K_{0}\right) \subset C_{0}$ for $m \leqq j$ $\leqq 2 m-1$. Then $f$ has a fixed point in $C_{0}$.

Proof. Let $A=\bigcup_{j=0}^{m-1} f^{j}\left(K_{0}\right)$. Then $A$ is a closed subcomplex of $K$ and $f(A) \subset A$, since $f^{m}\left(K_{0}\right) \subset C_{0} \subset A$. Furthermore, $f^{m}(A) \subset C_{0}$, a closed, bounded, acyclic subset of $A$. By Lemma $1, f$ has a fixed point in $A$, and, since $f^{m}(A) \subset C_{0}$, this fixed point must be in $C_{0}$.

LEMMA 3. In a Banach space $X$, let $f$ be a uniformly continuous mapping of a set $K$ into itself. For any given integer $m>0$ and any $\varepsilon>0$ there exists $\delta=\delta(\varepsilon)>0$ such that, if $g: D \rightarrow D$ is a self-mapping of the subset $D \subset K$ and $\|g(x)-f(x)\|<\delta$ on $D$, then $\left\|g^{j}(x)-f^{j}(x)\right\|<\varepsilon$ on $D$ for $1 \leqq j \leqq m$.

Proof. Obviously the statement is true for $m=1$. Assume it true for $1,2, \ldots$, $m-1$. Then

$$
\left\|g^{m}(x)-f^{m}(x)\right\| \leqq\left\|g\left(g^{m-1}(x)\right)-f\left(g^{m-1}(x)\right)\right\|+\left\|f\left(g^{m-1}(x)\right)-f\left(f^{m-1}(x)\right)\right\| .
$$

If we choose $\delta$ so that $\|g(x)-f(x)\|<\varepsilon / 2$ on $D,\left\|g^{j}(x)-f^{j}(x)\right\|<\varepsilon$ for $2 \leqq j \leqq m-1$, and so that $\left\|g^{m-1}(x)-f^{m-1}(x)\right\|<\eta$, where $\|f(x)-f(y)\|<\varepsilon / 2$ whenever $\|x-y\|<\eta$, then the result follows.

The fourth lemma is a restatement of Lemma II.7 of [6]. (This lemma may also be taken as a special case of Lemma 9, Dugundji's extension theorem.)

LEMMA 4 (GRANAS). There exists a retraction of any Banach space onto any closed, convex, separable subset of itself. In particular, there exists a retraction of a Banach space onto any compact, convex subset of itself.

LEMmA 5. Let $X$ be a finite-dimensional linear topological space and let $S_{0} \subset S_{1} \subset S_{2}$ be bounded convex sets of $X$ such that $S_{0}$ and $S_{2}$ are closed and $S_{1}$ is a neighborhood of $S_{0}$, relative to $S_{2}$. Let $f: S_{2} \rightarrow X$ be a continuous map such that for some integer $m>0$ we have

$$
f^{j}\left(S_{1}\right) \subset S_{2}, \quad 1 \leqq j \leqq m-1,
$$


and

$$
f^{j}\left(S_{1}\right) \subset S_{0}, \quad m \leqq j \leqq 2 m-1 .
$$

Then $f$ has a fixed point in $S_{0}$.

Proof. We may assume that $f\left(S_{2}\right) \subset S_{2}$, since if this is not the case we can consider a new map $\bar{f}=r f(x)$ where $r$ is a retraction of $X$ onto $S_{2}$, which exists by Lemma 4. It is clear that (1) and (2) are satisfied for $\bar{f}$ and that any fixed point of $\bar{f}$ in $S_{0}$ is a fixed point of $f$.

Now let $T: K_{\delta} \rightarrow S_{2}$ be a triangulation of $S_{2}$ of mesh $\delta$. Then for some barycentric subdivision $B_{r}\left(K_{\delta}\right)$, there exists a simplicial approximation $\bar{g}: B_{r}\left(K_{\delta}\right) \rightarrow K_{\delta}$ to the map $f$. Let $g: S_{2} \rightarrow S_{2}$ be $\bar{g}$ transferred back to $S_{2}$. Then we have for $x \in S_{2}$,

$$
\|f(x)-g(x)\| \leqq\left\|f(x)-f\left(v_{i}\right)\right\|+\left\|f\left(v_{i}\right)-g\left(v_{i}\right)\right\|+\left\|g\left(v_{i}\right)-g(x)\right\|,
$$

where $v_{i}$ is some vertex of a simplex of $B_{r}\left(K_{\delta}\right)$ (identified with $S_{2}$ ) containing $x$. Now $\left\|f\left(v_{i}\right)-g\left(v_{i}\right)\right\|<\delta$ and $g\left\|\left(v_{i}\right)-g(x)\right\|<\delta$, by the definition of simplicial approximation, and, since $\left\|x-v_{i}\right\|<\delta$, $\sup _{x \in S_{2}}\left\|f(x)-f\left(v_{i}\right)\right\| \rightarrow 0$ as $\delta \rightarrow 0$, by the uniform continuity of $f$. Thus

$$
\eta=\sup _{x \in S_{2}}\|f(x)-g(x)\| \rightarrow 0 \quad \text { as } \delta \rightarrow 0 .
$$

Then by Lemma 3,

$$
\eta_{1}=\sup \left\{\left\|f^{j}(x)-g^{j}(x)\right\|: x \in S_{2}, j \in[1,2 m]\right\} \rightarrow 0 \quad \text { as } \delta \rightarrow 0 .
$$

Since $S_{1}$ is a relative neighborhood of $S_{0}$, we have for some $\varepsilon>0$, that $N_{\varepsilon}\left(S_{0}\right)$ $\cap S_{2} \subset S_{1}$. Since $f^{j}\left(S_{1}\right) \subset S_{0}$ for $m \leqq j \leqq 2 m-1$, by hypothesis (2), it is clear that

$$
g^{j}\left(S_{1}\right) \subset \mathrm{Cl}\left(N_{n_{1}}\left(S_{0}\right)\right) \cap S_{2}
$$

for $m \leqq j \leqq 2 m-1$, by the definition of $\eta_{1}$ above. Let $S_{1}^{\prime}=\mathrm{Cl}\left(N_{\varepsilon / 2}\left(S_{0}\right)\right) \cap S_{2}$ and let $K_{1}^{\prime}$ be the subcomplex of $K_{\delta}$ consisting of those simplexes $\sigma$ such that $\sigma \cap S_{1}^{\prime} \neq \varnothing$. Then $S_{1}^{\prime} \subset K_{1}^{\prime}$, and it is also clear that if $\delta<\varepsilon / 2$ then $K_{1}^{\prime} \subset S_{1}$. Furthermore, if $\delta$ is also chosen so that $\eta_{1}<\varepsilon / 2$, then we have that

$$
g^{j}\left(K_{1}^{\prime}\right) \subset g^{j}\left(S_{1}^{\prime}\right) \subset \mathrm{Cl}\left(N_{\eta_{1}}\left(S_{0}\right)\right) \cap S_{2} \subset S_{1}^{\prime} .
$$

Applying Lemma 2 to the complex $K_{\delta}$, the subcomplex $K_{1}^{\prime}$, and the acyclic subset (because of its convexity) $S_{1}^{\prime}$, we see that $g$ has a fixed point in $S_{1}^{\prime}$, and in fact in $\mathrm{Cl}\left(N_{\eta_{1}}\left(S_{0}\right)\right) \cap S_{2}$.

Now let $\left\{\delta_{n}\right\}$ be a null sequence of $\delta$ 's for which the above construction is possible, let $\left\{g_{n}\right\}$ be the corresponding $g$ 's, and let $x_{n}$ be a fixed point of $g_{n}$, as found above. By the compactness of $S_{2}$, there exists a subsequence $x_{n(i)} \rightarrow x_{0}$. Then $f\left(x_{0}\right)=\lim g_{n(i)}\left(x_{n(i)}\right)=\lim x_{n(i)}=x_{0}$. Furthermore since $x_{n(i)} \in \mathrm{Cl}\left(N_{n_{1}}\left(S_{0}\right)\right)$ and $\eta_{1} \rightarrow 0$ as $\delta \rightarrow 0$, we must have $x_{0} \in S_{0}$. This completes the proof. 
THeOREM 6. Let $S_{0} \subset S_{1} \subset S_{2}$ be convex subsets of the Banach space $X$, with $S_{0}$ and $S_{2}$ compact and $S_{1}$ open relative to $S_{2}$. Let $f: S_{2} \rightarrow X$ be a continuous mapping such that, for some integer $m>0$,

$$
f^{j}\left(S_{1}\right) \subset S_{2}, \quad 1 \leqq j \leqq m-1,
$$

and

$$
f^{j}\left(S_{1}\right) \subset S_{0}, \quad m \leqq j \leqq 2 m-1 .
$$

Then $f$ has a fixed point in $S_{0}$.

Proof. We may assume that $f\left(S_{2}\right) \subset S_{2}$, since if this is not the case then by Lemma 4 , there exists a retraction $r: X \rightarrow S_{2}$. We may then define a new map $\bar{f}=r f$ which has properties (1) and (2) and whose fixed points in $S_{0}$ are also fixed points of $f$.

Since $S_{1}$ is open in $S_{2}$ and $S_{0}$ is compact, there exists $\varepsilon>0$ such that $N_{\varepsilon}\left(S_{0}\right)$ $\cap S_{2} \subset S_{1}$. Now by Lemma 3 there exists $\eta>0$ such that for any map $g$ defined on a subset $D$ of $S_{2}$ we have $\left\|g^{j}(x)-f^{j}(x)\right\|<\varepsilon / 2$ for $1 \leqq j \leqq 2 m$ and for all $x \in D$ whenever $\|g(x)-f(x)\|<\eta$ for all $x \in D$.

Let $\left\{x_{i}\right\}$ be a finite collection of points in $S_{2}$, with some $x_{j} \in S_{0}$, such that for any $x \in S_{2}$ there exists an $x_{i}$ with $\left\|x-x_{i}\right\|<\eta / 5$. Let $H$ be the finite-dimensional linear manifold generated by $\left\{x_{i}\right\}$. Let $R_{0}=S_{0} \cap H, R_{1}=S_{1} \cap H$, and $R_{2}=S_{2} \cap H$. Then $R_{0}, R_{1}$, and $R_{2}$ are nonempty convex sets in $H$. Therefore, there exists a triangulation $T: K \rightarrow R_{2}$ for some complex $K$. Since $f$ is uniformly continuous on $R_{2}$, there exists $\delta>0$ such that $\|f(x)-f(y)\|<\eta / 5$ whenever $\|x-y\|<\delta$. We may assume that the mesh of the triangulation $T$ is less than $\delta$, since a barycentric subdivision will give this.

Define a map $g: R_{2} \rightarrow R_{2}$ as follows. For each vertex $v \in R_{2}$ of the triangulation let $g(v)$ be some $x_{i}$ such that $\left\|x_{i}-f(v)\right\|<\eta / 5$. Extend $g$ to all of $R_{2}$ by the rule: if $x \in R_{2}$ and $T^{-1}(x)=\sum \alpha_{i} T^{-1}\left(v_{i}\right)$, then $g(x)=\sum \alpha_{i} g\left(v_{i}\right)$.

Now if $v_{i}$ and $v_{j}$ are vertices of a common simplex in $R_{2}$, then we have

$$
\begin{aligned}
\left\|g\left(v_{i}\right)-g\left(v_{j}\right)\right\| & \leqq\left\|g\left(v_{i}\right)-f\left(v_{i}\right)\right\|+\left\|f\left(v_{i}\right)-f\left(v_{j}\right)\right\|+\left\|f\left(v_{j}\right)-g\left(v_{j}\right)\right\| \\
& <\eta / 5+\eta / 5+\eta / 5=3 \eta / 5 .
\end{aligned}
$$

Thus if $x$ and $y$ are any points of $R_{2}$ contained in the same simplex of $K$, then $\|g(x)-g(y)\|<3 \eta / 5$ also, since the distance between $g(x)$ and $g(y)$ is no greater than the maximum distance between the $g$-images of any two vertices of the containing simplex. Therefore we have, for any $x \in R_{2}$,

$$
\begin{aligned}
\|f(x)-g(x)\| & \leqq\|f(x)-f(u)\|+\|f(u)-g(u)\|+\|g(u)-g(x)\| \\
& <\eta / 5+\eta / 5+3 \eta / 5=\eta
\end{aligned}
$$

where $u$ is any vertex of a simplex containing $x$.

From the above, plus the fact that $f^{j}\left(S_{1}\right) \subset S_{0}$ for $m \leqq j \leqq 2 m-1$, we see that

$$
g^{j}\left(R_{1}\right) \subset N_{\varepsilon / 2}\left(f^{j}\left(R_{1}\right)\right) \subset N_{\varepsilon / 2}\left(f^{j}\left(S_{1}\right)\right) \subset N_{\varepsilon / 2}\left(S_{0}\right) \text { for } m \leqq j \leqq 2 m-1 \text {. }
$$


But $g: R_{2} \rightarrow R_{2}$, and so we have

$$
g^{j}\left(R_{1}\right) \subset N_{\varepsilon / 2}\left(S_{0}\right) \cap R_{2}=N_{\varepsilon / 2}\left(R_{0}\right) \cap R_{2} \text { for } m \leqq j \leqq 2 m-1 .
$$

Let $R_{0}^{\prime}=N_{\varepsilon / 2}\left(R_{0}\right) \cap R_{2}$. Then $R_{0}^{\prime}$ is closed and $N_{\varepsilon / 2}\left(R_{0}^{\prime}\right) \cap H \subset R_{1}$. But $g^{j}\left(R_{1}\right) \subset R_{0}^{\prime}$, for $m \leqq j \leqq 2 m-1$, and so by the previous lemma $g$ has a fixed point in $R_{0}^{\prime}$.

Now let $\left\{\varepsilon_{n}\right\}$ be a null sequence of $\varepsilon$ 's as considered above, and let $\left\{g_{n}\right\}$ be the corresponding maps with fixed points $\left\{x_{n}\right\}$ respectively. By the compactness of $S_{2}$ there exists a subsequence $x_{n(i)} \rightarrow x_{0}$. By the reasoning of Lemma $5, f\left(x_{0}\right)=x_{0}$ and $x_{0} \in S_{0}$. This completes the proof.

THEOREM 7. Let $S_{0} \subset S_{1} \subset S_{2}$ be convex subsets of the Banach space $X$, with $S_{0}$ and $S_{2}$ closed and $S_{1}$ open relative to $S_{2}$. Let $f: S_{2} \rightarrow X$ be a continuous, compact mapping such that for some integer $m>0$,

$$
f^{j}\left(S_{1}\right) \subset S_{2}, \quad 1 \leqq j \leqq m-1,
$$

and

$$
f^{j}\left(S_{1}\right) \subset S_{0}, \quad m \leqq j \leqq 2 m-1 .
$$

Then $f$ has a fixed point in $S_{0}$.

Proof. Let $S_{2}^{\prime}=h\left(f\left(S_{2}\right)\right)$, where $h$ is the convex closure operator. Let $R_{0}=S_{0}$ $\cap S_{2}^{\prime}, R_{1}=S_{1} \cap S_{2}^{\prime}$, and $R_{2}=S_{2} \cap S_{2}^{\prime}$. Then $R_{0}$ and $R_{2}$ are compact, and $R_{1}$ is open relative to $R_{2}$. Furthermore, we may define $\bar{f}: R_{2} \rightarrow R_{2}$ by $\bar{f}=r f$, where $r$ is any retraction of $X$ onto $R_{2}$, which exists by Lemma 4. Then $\bar{f}^{j}\left(R_{1}\right) \subset R_{2}$ for $1 \leqq j \leqq m-1$ and $\bar{f}^{j}\left(R_{1}\right) \subset R_{0}$ for $m \leqq j \leqq 2 m-1$, since $\bar{f}^{j}=f^{j}$ on $R_{1}$. Applying Theorem 6 to $R_{0}, R_{1}$, and $R_{2}$, we see that $\bar{f}$, and hence $f$, has a fixed point in $R_{0} \subset S_{0}$.

Corollary 8. Let $S_{0} \subset S_{1} \subset S_{2}$ be convex subsets of the Banach space $X$, with $S_{0}$ and $S_{2}$ closed and $S_{1}$ open relative to $S_{2}$. Let $f: S_{2} \rightarrow X$ be a continuous compact mapping such that, for some integer $m>0$,

$$
\begin{array}{ll}
f^{j}\left(S_{0}\right) \subset S_{1}, & 1 \leqq j \leqq m-1, \\
f^{j}\left(S_{1}\right) \subset S_{2}, & 1 \leqq j \leqq m-1,
\end{array}
$$

and

$$
f^{m}\left(S_{1}\right) \subset S_{0}
$$

Then $f$ has a fixed point in $S_{0}$.

Proof. By (1) and (3) we have that $f^{j}\left(S_{1}\right) \subset S_{0}$ for $2 m \leqq j \leqq 4 m-1$. Thus Theorem 7 applies.

Corollary 8 is very similar to Browder's theorem in [1].

The next lemma is a statement of an extension theorem proved by J. Dugundji in [5]. 
Lemma 9 (Dugundu). Let $X$ be an arbitrary metric space, $A$ a closed subset of $X$, $L$ a locally convex linear topological space, and $f: A \rightarrow L$ a continuous map. Then there exists an extension $F: X \rightarrow L$ of $f$. Furthermore, $F(X) \subset[$ convex hull of $f(A)]$

The next theorem shows that the set $S_{2}$ of Theorems 6 and 7 is superfluous in some sense.

THEOREM 10. Let $S_{0} \subset S_{1}$ be convex subsets of the Banach space $X$ with $S_{0}$ closed and $S_{1}$ open. Let $f$ be a compact continuous mapping defined on some closed subset $D$ of $X$ such that $f^{j}\left(S_{1}\right) \subset D$ for all $j \geqq 0$ and such that, for some integer $m>0$, $\bigcup_{j=m}^{2 m-1} f^{j}\left(S_{1}\right) \subset S_{0}$. Then $f$ has a fixed point in $S_{0}$.

Proof. Note that the hypotheses imply $f^{j}\left(S_{1}\right) \subset S_{0}$ for $j \geqq m$. Let

$$
A=\mathrm{Cl}\left(\bigcup_{j=0}^{m} f^{j}\left(S_{1}\right)\right)
$$

Then $f(A) \subset A$. Furthermore, by Dugundji's theorem there exists an extension of the map $f \mid A$ to a map $\bar{f}: X \rightarrow X$. Since $\bar{f}|A=f| A$, we apply Theorem 7 to show that $\bar{f}$, and hence $f$, has a fixed point in $S_{0}$.

Next we show that the condition $\bigcup_{j=m}^{2 m-1} f^{j}\left(S_{1}\right) \subset S_{0}$ is also unnecessarily strong, and, in fact, that only two iterates of $f$ need take $S_{1}$ into $S_{0}$ to produce a fixed point for $f$ (Corollary 13).

LEMMA 11. Let $N$ be any set of positive integers with $c$ the greatest common factor of all $n \in N$. Then there exists a positive integer $m$ and a subset $\left\{n_{i}\right\}_{i=1}^{k} \subset N$ such that all integers of the form $c j$, where $m \leqq j \leqq 2 m$, can be written as

$$
c j=\sum_{i=1}^{k} a_{i} n_{i}
$$

where the $a_{i}$ 's are nonnegative integers (dependent on $j$ ).

Proof. The lemma is well known and the proof may be found in a number of good books. See, for example, Finite Markov Chains by Kemeny and Snell, Van Nostrand, Princeton, N. J., 1960, pp. 6-7.

THEOREM 12. Let $S_{0} \subset S_{1} \subset S_{2}$ be convex subsets of the Banach space $X$ with $S_{0}$ and $S_{2}$ closed and $S_{1}$ open relative to $S_{2}$. Let $f: S_{2} \rightarrow X$ be a continuous, compact mapping such that, for some set of positive integers $N$,

$$
f^{j}\left(S_{1}\right) \subset S_{2}, \quad \text { for all } j \geqq 1,
$$

and

$$
f^{j}\left(S_{1}\right) \subset S_{0}, \quad j \in N .
$$

Let $c=g c f(N)$. Then $f^{c}$ has a fixed point in $S_{0}$. 
Proof. By Lemma 11, there exists a subset $\left\{n_{i}\right\}_{i=1}^{k} \subset N$ such that for some positive integer $m$ we may write $j c=\sum_{i=1}^{1} a_{i} n_{i}$ for each $j$ such that $m \leqq j \leqq 2 m$, where $a_{i} \geqq 0$ and the $a_{i}$ depend on $j$. Thus

$$
\begin{aligned}
f^{j c}\left(S_{1}\right) & =f^{a_{1} n_{1}}\left(f^{a_{2} n_{2}}\left(\cdots\left(f^{a_{k} n_{k}}\left(S_{1}\right)\right) \cdots\right)\right. \\
& \subset f^{a_{1} n_{1}}\left(f^{a_{2} n_{2}}\left(\cdots\left(f^{a_{k-1} n_{k-1}}\left(S_{0}\right)\right) \cdots\right)\right. \\
& \subset \cdots \subset \cdots \subset S_{0} .
\end{aligned}
$$

Applying Theorem 7 completes the proof.

Corollary 13. Let $S_{0} \subset S_{1} \subset S_{2}$ be convex subsets of the Banach space $X$ with $S_{0}$ and $S_{2}$ closed and $S_{1}$ open relative to $S_{2}$. Let $f: S_{2} \rightarrow X$ be a continuous, compact mapping such that, for some integer $m>0$,

$$
\begin{gathered}
f^{j}\left(S_{1}\right) \subset S_{2}, \quad 1 \leqq j \leqq m, \\
f^{m}\left(S_{1}\right) \cup f^{m+1}\left(S_{1}\right) \subset S_{0} .
\end{gathered}
$$

Then $f$ has a fixed point in $S_{0}$.

Proof. Since $f^{j}\left(S_{1}\right) \subset S_{2}$ for $1 \leqq j \leqq m$ and $f^{m}\left(S_{1}\right) \subset S_{0} \subset S_{1}$, the iterates of $f$ are well defined on $S_{1}$ for all $j \geqq 1$. Applying Theorem 12 gives us the desired result.

B. Ejective and nonejective fixed points. Closely related to the theorems of $\S \mathrm{A}$ is the subject of a compact mapping of a convex set into itself, with fixed points whose neighborhoods eventually (in some sense) map outside of themselves. To be more precise, we introduce the terms "repulsive" and "ejective" fixed point, defined by Browder in [2] and [3]. Let $C$ be a subset of the Banach space $X, f$ a continuous mapping of $C$ into itself. If $x_{0}$ is a fixed point of $f, x_{0}$ is said to be repulsive if there exists a neighborhood $U$ of $x_{0}$ in $C$ such that for every $x \in C-\left\{x_{0}\right\}$ there exists an integer $J(x)$ such that $f^{j}(x) \in C-U$ for $j \geqq J(x)$. Also, $x_{0}$ is said to be an ejective fixed point of $f$ if there exists a (relative) neighborhood $U$ of $x_{0}$ in $C$ such that for any $x \in U-\left\{x_{0}\right\}$ there exists a positive integer $k(x)$ such that $f^{k(x)}(x)$ $\in C-U$.

Now let $f$ be a continuous mapping of the infinite-dimensional, compact, convex set $C$ into itself. In [2] Browder proved that $f$ has a nonrepulsive fixed point and in [3] the stronger result that $f$ has a nonejective fixed point.

We would like to use the results of [3] to prove a theorem about mappings which take each point of the space, on some iterate, into a given bounded set. To start we have the following:

THEOREM 14. Let $C$ be an infinite-dimensional closed, convex set in the Banach space $X$ and $f$ a compact mapping of $C$ into itself. Then $f$ has a nonejective fixed point.

Proof. Let $\left\{x_{n}\right\}$ be an infinite-dimensional sequence of points in $C$ such that $d\left(x_{n}, f(C)\right)<2^{-n}$. Clearly such a sequence exists. Let

$$
C_{1}=h\left(\bigcup\left\{x_{n}\right\} \cup f(C)\right) \subset C,
$$


where $h$ denotes the convex closure operator. Then $\cup\left\{x_{n}\right\} \cup f(C)$ is clearly compact, so that $C_{1}$ is also compact, and we have

$$
f\left(C_{1}\right) \subset f(C) \subset C_{1} .
$$

Thus, since $C_{1}$ is infinite-dimensional, by Browder's theorem $f$ has a nonejective fixed point in $C_{1}$. But by the definition of ejectivity, a fixed point of $f$ which is nonejective in $C_{1}$ is also nonejective in $C$. This proves the theorem.

THEOREM 15. Let $X$ be an infinite-dimensional Banach space and $f$ a completely continuous mapping of $X$ into itself such that for each $K>0$ there exists $k>0$ such that $\|f(x)\|>K$ whenever $\|x\|>k$. Suppose also that there exists a bounded set $E$ such that for each $x \in X$ there is a positive integer $m=m(x)$ with $f^{m}(x) \in E$. Then $f$ has a nonejective fixed point in $E$.

Proof. First we show that $X-f(X)$ contains an open set. Let $B_{a}$ denote the open ball about the origin of radius $a$. Given $K>0$, we can find $k>0$ such that

$$
f\left(X-\mathrm{Cl}\left(B_{k}\right)\right) \subset X-\mathrm{Cl}\left(B_{K}\right) .
$$

Furthermore, $f\left(\mathrm{Cl}\left(B_{k}\right)\right)$ is a compact set, since $f$ is completely continuous, and so $B_{K}-f\left(\mathrm{Cl}\left(B_{k}\right)\right)$ is a nonvoid open set. Thus, since $B_{K}-f\left(\mathrm{Cl}\left(B_{k}\right)\right) \subset X-f(X)$, there is an open set contained in $X-f(X)$.

Let $x_{0}$ be an interior point of $X-f(X)$, and we may assume without loss of generality that $x_{0}=0$. Then there exists $\varepsilon$ such that $N_{\varepsilon}(0)=B_{\varepsilon} \subset X-f(X)$. Thus $f$ maps $X-B_{\varepsilon}$ into itself. Also, there exists $B_{r}$ containing $B_{\varepsilon}$ and $E$, so that for any $x \in X$ there is a positive integer $m=m(x)$ such that $f^{m}(x) \in B_{r}$. Now let the involution $\varphi: X-\{0\} \rightarrow X-\{0\}$ be defined by $\varphi(x)=x /\|x\|^{2}$. Let $g: X-\{0\} \rightarrow X-\{0\}$ be defined by $g=\varphi f \varphi$. Extend $g$ to all of $X$ by defining $g(0)=0$. Now $g$ is continuous on $X-\{0\}$, since $f$ is continuous, and $g$ is continuous at 0 , since for a given $\eta>0$, there exists $\delta>0$ such that $\|f(x)\|>1 / \eta$ whenever $\|x\| \geqq 1 / \delta$. Thus $\|g(x)\|<\eta$ whenever $\|x\|<\delta$.

Also, since $f\left(X-B_{\varepsilon}\right) \subset X-B_{\varepsilon}$, we have $g\left(\mathrm{Cl}\left(B_{1 / \varepsilon}\right)\right) \subset \mathrm{Cl}\left(B_{1 / \varepsilon}\right)$.

Next we show that $g \mid \mathrm{Cl}\left(B_{1 / \varepsilon}\right)$ is compact. For if $\left\{x_{n}\right\}$ is any sequence of points in $\mathrm{Cl}\left(B_{1 / \varepsilon}\right)$, then if $\left\{x_{n}\right\}$ has 0 as a limit point so does $\left\{g\left(x_{n}\right)\right\}$.

If $\left\{x_{n}\right\}$ does not have 0 as a limit point, then the $x_{n}$ 's are bounded away from 0 , so that $\left\{\varphi\left(x_{n}\right)\right\}$ is a bounded sequence. Thus $\left\{f \varphi\left(x_{n}\right)\right\}$ has a limit point (in $X-B_{\varepsilon}$ ), by the complete continuity of $f$, so that $\left\{g\left(x_{n}\right)\right\}$ also has a limit point. This shows that every infinite set of points in $g\left(\mathrm{Cl}\left(B_{1 / \varepsilon}\right)\right)$ has a limit point, and so $g \mid \mathrm{Cl}\left(B_{1 / \varepsilon}\right)$ is a compact mapping.

Finally, 0 is an ejective fixed point of $g \mid \mathrm{Cl}\left(B_{1 / \varepsilon}\right)$. For if $x \in B_{1 / r}, x \neq 0$, then for some $m>0$ we have $f^{m} \varphi(x) \in E \subset B_{r}$. Thus $g^{m}(x)=\varphi f^{m} \varphi(x) \in X-B_{1 / r}$.

Applying Theorem 14, we see that $g \mid B_{1 / \varepsilon}$ has a nonejective fixed point $x_{0}$ in $\mathrm{Cl}\left(B_{1 / \varepsilon}\right)$. Now $x_{0} \neq 0$, since 0 is ejective, and if $x_{0}$ is nonejective under $g$ then $\varphi\left(x_{0}\right)$ is nonejective under $f$. Thus $\varphi\left(x_{0}\right)$ is a nonejective fixed point of $f$ which must lie in $E$, since $f^{m} \varphi\left(x_{0}\right) \in E$ for some $m>0$. This completes the proof. 
If considerations of ejectivity of the fixed point can be neglected then a much stronger result can be obtained, as shown by the following theorem.

THEOREM 16. Let $f$ be a completely continuous mapping of the Banach space $X$ into itself. Suppose that there exists a bounded set $E$ such that for each $x \in X$ there exists $m=m(x)$ such that $f^{m}(x) \in E$. Then $f$ has a fixed point in $E$.

Proof. Let $T$ be any closed ball containing $E$ in its interior and let $R=\mathrm{Cl}(f(T))$, a compact set. From the hypotheses, for each $x \in R$ there exists $m=m(x)$ such that $f^{m}(x) \in E$. But since $E \subset T^{0}$ there exists $\varepsilon=\varepsilon(x)$ such that $f^{m}\left(N_{\varepsilon}(x)\right) \subset T$. Assign such an $m(x)$ and $\varepsilon(x)$ to each $x \in R$. Then the collection of open sets $\left\{N_{\varepsilon(x)}(x)\right\}$ covers $R$, and by the compactness of $R$ there exists a finite set $\left\{x_{1}, x_{2}, \ldots, x_{n}\right\}$ of points in $R$, with associated sets $\left\{m_{1}, m_{2}, \ldots, m_{n}\right\}$ and $\left\{\varepsilon_{1}, \varepsilon_{2}, \ldots, \varepsilon_{n}\right\}$, such that

$$
f^{m_{i}}\left(N_{\varepsilon_{i}}\left(x_{i}\right) \cap R\right) \subset T \text { and } R \subset \bigcup_{i=1}^{n} N_{\varepsilon_{i}}\left(x_{i}\right) \text {. }
$$

Let $m_{0}=\max \left\{m_{i}\right\}$ and let $S_{0}=h\left(\bigcup_{j=0}^{m_{0}+1} f^{j}(T)\right)$, where $h$ is the convex closure operator. Let $S_{1}=N_{\eta}\left(S_{0}\right)$, where $\eta$ is any positive number.

For any $x \in T$ it is easy to show that $f^{j}(x) \in S_{0}$ for any $j \geqq 0$. For if $x \in T$, assume $f^{j}(x) \in S_{0}$ for $j \leqq j_{0}$. If $j_{0} \leqq m_{0}$ then it is obvious that $f^{j_{0}+1}(x) \in S_{0}$, by the definition of $S_{0}$. If $j_{0}>m_{0}$, then $f(x) \in R$ and hence $f^{k+1}(x) \in T$ for some $k \leqq m_{0}$. Thus

$$
f^{j}(x)=f^{j_{0}-k-1}\left(f^{k+1}(x)\right) \in f^{j_{0}-k-1}(T),
$$

and the result holds by induction.

Now it may be shown in the same way as above that there exists some positive integer $m^{\prime}$ such that, for each $x \in S_{1}, f^{j}(x) \in T$ for some $j \leqq m^{\prime}$. But then $f^{j}(x) \in S_{0}$ for all $j \geqq m^{\prime}$ by the above. Letting $S_{2}=h\left(\bigcup_{j=0}^{m^{\prime}} f^{j}\left(S_{1}\right)\right)$ and applying Theorem 7 to $S_{0}, S_{1}$, and $S_{2}$, we see that $f$ has a fixed point in $S_{0}$. But for any $x \in S_{0}, f^{j}(x) \in E$ for some $j>0$. Thus this fixed point must lie in $E$, thereby proving the theorem.

C. Extensions to flows. Many of the previous theorems have analogues in the theory of flows. In this case, proof of the existence of a common fixed point of all the members of the flow, a so-called stationary point, is the desired result to be obtained.

Let $\left\{T_{s}: s \in S\right\}$ be a set of continuous mappings of a subset $Y$ of the Banach space $X$ into itself, where $S$ is a commutative topological semigroup with identity element 0 such that

and

$$
T_{0}(x)=x, \quad x \in X,
$$

$$
\begin{aligned}
T_{s}\left(T_{t}(x)\right) & =T_{t}\left(T_{s}(x)\right) \\
& =T_{t+s}(x), \quad x \in X ; \quad s, t \in S,
\end{aligned}
$$

and satisfying the continuity condition that for each $t \in S$

$$
\sup \left\{\left\|T_{t}(x)-T_{s}(x)\right\|: x \in Y\right\} \rightarrow 0 \text { as } s \rightarrow t .
$$


Then $\left\{T_{s}: s \in S\right\}$ is called an $S$-semigroup of operators on $Y$. We shall consider only the case where $S=R^{+}$, the nonnegative real numbers with the usual topology. In this case, $\left\{T_{s}: s \in R^{+}\right\}$is called a flow.

The following theorems extend the previous results to flows $\left({ }^{2}\right)$.

TheOREM 17. Let $\left\{T_{s}: s \in R^{+}\right\}$be a flow on the subset $Y$ of the Banach space $X$. Let $C_{0} \subset C_{1} \subset C_{2}$ be convex subsets of $Y$ such that $C_{0}$ and $C_{2}$ are compact and $C_{1}$ is a neighborhood of $C_{0}$ relative to $C_{2}$. Suppose further that for some closed interval $[a, b]$ with $0 \leqq a<b$ we have

$$
\begin{array}{ll}
T_{s}\left(C_{1}\right) \subset C_{2}, & s \in[0, a], \\
T_{s}\left(C_{1}\right) \subset C_{0}, & s \in[a, b] .
\end{array}
$$

Then there exists a point $x_{0} \in C_{0}$ such that $T_{s}\left(x_{0}\right)=x_{0}$ for all $s \in R^{+}$.

Proof. For $K=2 /(b-a)$, we have that for any integer $k>K$ there exists an integer $m$ such that $a<m / k<(m+1) / k<b$. Thus $T_{m / k}\left(C_{1}\right) \subset C_{0}$ and $T_{(m+1) / k}\left(C_{1}\right) \subset C_{0}$. That is, $T_{1 / k}^{m}\left(C_{1}\right) \subset C_{0}$ and $T_{1 / k}^{m+1}\left(C_{1}\right) \subset C_{0}$. Thus by Corollary $13 T_{1 / k}$ has a fixed point $x_{k} \in C_{0}$. Since $C_{0}$ is compact, there exists a limit point $x_{0}$ of the set $\left\{x_{n}\right\}$ and a subsequence of $\left\{x_{n}\right\}$, which we denote by $\left\{y_{n}\right\}$, such that $y_{n} \rightarrow x_{0}$.

Now let $T_{s}$ be any member of the flow. For any interger $k>0$, there exists an integer $j \geqq 0$ such that $|s-j| k \mid<1 / k$. Let $g_{k}=T_{j \mid k}$. Then $g_{k}\left(x_{k}\right)=x_{k}$ and $g_{k} \rightarrow T_{s}$. For each $y_{n}=x_{m_{n}}$ of the previously determined subsequence, let $f_{n}=g_{m_{n}}$. Thus $y_{n} \rightarrow x_{0}, f_{n}\left(y_{n}\right)=y_{n}$, and $f_{n} \rightarrow T_{s}$. From this we have

$$
T_{s}\left(x_{0}\right)=\lim _{n \rightarrow \infty} f_{n}\left(y_{n}\right)=\lim _{n \rightarrow \infty} y_{n}=x_{0}
$$

This completes the proof.

Even this result is not the strongest possible. We may also have $b=a$, that is, $T_{a}\left(C_{1}\right) \subset C_{0}$ only, and a common fixed point will still exist.

THEOREM 18. Let $\left\{T_{s}: s \in R^{+}\right\}$be a flow on the subset $Y$ of the Banach space $X$. Let $C_{0} \subset C_{1} \subset C_{2}$ be convex subsets of $Y$ such that $C_{0}$ and $C_{2}$ are compact and $C_{1}$ is a neighborhood of $C_{0}$ relative to $C_{2}$. Suppose further that for some a>0 we have

$$
\begin{aligned}
& T_{s}\left(C_{1}\right) \subset C_{2}, \quad s \in[0, a], \\
& T_{a}\left(C_{1}\right) \subset C_{0} .
\end{aligned}
$$

Then there exists a point $x_{0} \in C_{0}$ such that $T_{s}\left(x_{0}\right)=x_{0}$ for all $s \in R^{+}$.

Proof. Since $C_{0}$ is compact, there exists $\varepsilon>0$ such that $N_{\varepsilon}\left(C_{0}\right) \cap C_{2} \subset C_{1}$. For any $\eta<\varepsilon, \eta>0$, there exists $\delta>0$ such that $\left\|T_{s}(x)-T_{a}(x)\right\|<\eta$ whenever $|s-a| \leqq \delta$, for all $x \in Y$, by the continuity of the flow. Thus

$$
T_{s}\left(C_{1}\right) \subset \mathrm{Cl}\left(N_{\eta}\left(C_{0}\right) \cap C_{2}\right)=C_{0}^{\prime}
$$

(2) It has been brought to the author's attention by G. S. Jones that several of the results on flows presented here could also be obtained from the Schauder theorem, using a theorem on continuous continuation of mappings (cf. [6, Theorem V.2, p. 49]). 
for $s \in[a-\delta, a]$ and so there is a stationary point $z$ of the flow in $C_{0}^{\prime}$, by the previous theorem. Let $\left\{\eta_{n}\right\}$ be a null sequence, $C_{0}^{(n)}=N_{n_{n}}\left(C_{0}\right) \cap C_{2}$, and $z_{n}$ be the stationary point of the flow in $C_{0}^{(n)}$ found by the above. It is clear that the sequence $\left\{z_{n}\right\}$ has a limit point in $C_{0}$ which must also be a stationary point of the flow. This completes the proof.

An extension to flows is also possible for Browder's theorem on nonejective fixed points. If $\left\{T_{s}: s \in R^{+}\right\}$is a flow on the set $C$, we define $x_{0}$ to be an ejective stationary point of the flow if $T_{s}\left(x_{0}\right)=x_{0}$ for all $s \in R^{+}$and for some neighborhood $U$ of $x_{0}$ in $C$ we have that for each $x \in U-\left\{x_{0}\right\}$ there exists $s=s(x)>0$ such that $T_{s}(x) \in C-U$.

TheOREM 19. Let $\left\{T_{s}: s \in R^{+}\right\}$be a flow on the infinite-dimensional compact, convex set $C$ in the Banach space $X$. Then $\left\{T_{s}\right\}$ has a nonejective stationary point.

Proof. From Browder's theorem, each $T_{s}$ has a nonejective point in $C$. Let $\left\{T_{s_{n}}\right\}$ be a sequence of maps with $s_{n}>0, s_{n} \rightarrow 0$, and let $x_{n}$ be a nonejective fixed point of $T_{s_{n}}$. We may assume, without loss of generality, that $x_{n} \rightarrow x_{0}$. As shown in the proof of Theorem 17, $x_{0}$ is then a fixed point for all $T_{s}$.

Now suppose that $x_{0}$ is an ejective stationary point. By the definition of ejectivity, this means that there is a neighborhood $U$ of $x_{0}$ in $C$ such that for any neighborhood $U_{1} \subset U$ we have that for each $x \in U_{1}-\left\{x_{0}\right\}$ there exists $t=t(x)>0$ such that $T_{t}(x)$ $\in C-U_{1}$. Let $\varepsilon>0$ be such that $V=N_{\varepsilon}\left(x_{0}\right) \cap C \subset U$ and let $V_{1}=N_{\varepsilon / 2}\left(x_{0}\right) \cap C$. Then for each $x \in V_{1}-\left\{x_{0}\right\}$ there exists $t=t(x)>0$ such that $T_{t}(x) \in C-V$.

Consider any point of the previous sequence $x_{n} \in V_{1}$. Since $x_{n}$ is a nonejective fixed point of $T_{s_{n}}$, there exists some $z_{n} \in V_{1}$ such that $z_{n} \neq x_{0}$ and $T_{s_{n}}^{k}\left(z_{n}\right) \in V_{1}$ for all integers $k \geqq 0$. But by the ejectivity of $x_{0}$, there exists $t_{n}>0$ such that $T_{t_{n}}\left(z_{n}\right)$ $\in C-V$. Let $k \geqq 0$ be an integer such that

Then

$$
\left|k s_{n}-t_{n}\right|<s_{n} \text {, and } k s_{n} \leqq t_{n} .
$$

$$
T_{t_{n}}\left(z_{n}\right)=T_{t_{n}-k s_{n}}\left(T_{k s_{n}}\left(z_{n}\right)\right)=T_{t_{n}-k s_{n}}\left(y_{n}\right)
$$

where $y_{n} \in V_{1}$ and $T_{t_{n}-k s_{n}}\left(y_{n}\right) \in C-V$. Thus

$$
\left\|y_{n}-T_{t_{n}-k s_{n}}\left(y_{n}\right)\right\| \geqq \varepsilon / 2 .
$$

But by the continuity of the flow at $T_{0}$, there exists $\delta>0$ such that

$$
\left\|T_{t}(x)-x\right\|<\varepsilon / 2
$$

for all $t<\delta$, and all $x \in C$. Since $\lim _{n \rightarrow \infty} t_{n}-k s_{n}=0$, we have established a contradiction and the theorem is proved.

The final theorem on flows is an extension of Theorem 16. We shall consider only the finite-dimensional case, since it would seem to be difficult, if not impossible, to find a flow $\left\{T_{s}\right\}$ on an infinite-dimensional space such that $T_{s}$ is completely continuous for $s>0$ and $T_{0}$ is the identity. 
TheOREM 20. Let $X$ be a finite-dimensional Banach space and $\left\{T_{s}: s \in R^{+}\right\} a$ flow on $X$. Suppose there exists a bounded set $E$ such that for each $x \in X$ there exists $s=s(x)$ such that $T_{s}(x) \in E$. Then there is a stationary point of the flow in $E$.

Proof. Let $B_{r}$ be a closed ball about the origin of radius $r$ containing $E$. By the continuity of the flow at $T_{0}$, there exists $\delta>0$ such that $\left\|T_{t}(x)-x\right\|<r$ for $t<\delta$. Let $\left\{t_{n}\right\}$ be a null sequence with each $t_{n}<\delta$. For each $x \in X$, there exists $s=s(x)$ such that $T_{s}(x) \in E \subset B_{r}$. Let $k_{n}=k_{n}(x)$ be a nonnegative integer such that

$$
\left|k_{n} t_{n}-s\right|<t_{n}<\delta \text { and } k_{n} t_{n}<s .
$$

Since $T_{s}(x) \in B_{r}$ and

$$
\left\|T_{s}(x)-T_{k_{n} t_{n}}(x)\right\|=\left\|T_{s-k_{n} t_{n}}\left(T_{k_{n} t_{n}}(x)\right)-T_{k_{n} t_{n}}(x)\right\|<r,
$$

we have that $T_{k_{n} t_{n}}(x)=T_{t_{n}}^{k_{n}}(x) \in B_{2 r}$. Since such a $k_{n}$ exists for each $x$, we may apply Theorem 16 to show that $T_{t_{n}}$ has a fixed point $x_{n} \in E$. As shown in Theorem 17 , a limit point $x_{0}$ of the sequence $\left\{x_{n}\right\}$ is a stationary point of the flow. Since $T_{s_{0}}\left(x_{0}\right) \in E$ for some $s_{0}>0$, we must have $x_{0} \in E$, completing the proof.

D. Conjectures and examples. One feels that the "intermediate" set $S_{1}$ in Theorem 12 is not really necessary, so that the following might be conjectured:

Let $S_{0} \subset S_{2}$ be compact, convex subsets of the Banach space $X$ and $f$ a continuous map of $S_{2}$ into $X$ such that, for some set of positive integers $N$,

$$
f^{j}\left(S_{0}\right) \subset S_{2} \text { for all } j \geqq 1
$$

and

$$
f^{j}\left(S_{0}\right) \subset S_{0}, \quad j \in N .
$$

Let $c=g c f(N)$. Then $f^{c}$ has a fixed point in $S_{0}$.

This could be proved, for instance, if there exists a sequence of maps $\left\{f_{n}\right\}$, where $f_{n}: S_{2} \rightarrow X, f_{n} \rightarrow f$, and a sequence of intermediate convex sets $\left\{S_{1}^{n}\right\}$ such that $S_{0} \subset S_{1}^{n}, S_{1}^{n}$ is open in $S_{2}, f_{n}^{j}\left(S_{1}^{n}\right) \subset S_{2}$ for all $j \geqq 1, f_{n}^{j}\left(S_{1}^{n}\right) \subset S_{0}$ for $j \in N$, and $S_{1}^{n} \rightarrow S_{0}$. For then each $f_{n}^{c}$ has a fixed point in $S_{1}^{n}$ and a limit point of these points must lie in $S_{0}$ and be fixed under $f^{c}$.

Also, since we may show as in the proof of Theorem 12 that for some $m>0$ we have $f^{j c}\left(S_{0}\right) \subset S_{0}$ for $j \geqq m$, if there exists a retraction $r$ taking $\left[N_{\varepsilon}\left(S_{0}\right) \cap S_{2}\right]$ $\cup \bigcup_{j=1}^{m} f^{j c}\left(S_{0}\right)$ onto $\bigcup_{j=0} f^{j c}\left(S_{0}\right)$, then it is possible to define $\bar{f}=f^{c} r$ on $\left[N_{\varepsilon}\left(S_{0}\right) \cap S_{2}\right]$ $\cup \bigcup_{j=1}^{m} f^{j c}\left(S_{0}\right)$. By Dugundji's theorem, $\bar{f}$ may be extended to a map $g$ taking all of $S_{2}$ into itself. Letting $S_{1}=N_{\varepsilon}\left(S_{0}\right) \cap S_{2}$, we see that $g^{j}\left(S_{1}\right) \subset S_{0}$ for all $j \geqq m$, and so Theorem 6 applies.

A second conjecture which, if true, would prove the first is the following:

Let $S$ be a compact, convex subset of the Banach space $X$, and $f, g: S \rightarrow S$ be continuous, commuting maps. Then there exists a point $x_{0} \in S$ such that $f\left(x_{0}\right)$ $=g\left(x_{0}\right)$. 
For if $f$ satisfies the hypotheses of the first conjecture, then again there exists $m>0$ such that $f^{j c}\left(S_{0}\right) \subset S_{0}$ for $j \geqq m$. Thus $f^{m c}, f^{(m+1) c}: S_{0} \rightarrow S_{0}$ and so there exists $x_{0}$ such that

$$
f^{m c}\left(x_{0}\right)=f^{(m+1) c}\left(x_{0}\right)=f^{c}\left(f^{m c}\left(x_{0}\right)\right) ;
$$

thus $f^{m c}\left(x_{0}\right)$ is a fixed point of $f^{c}$.

The second conjecture is true if either $f$ or $g$ is a homeomorphism onto, for then we have that either $f^{-1} g$ or $g^{-1} f$ takes $S$ into itself and has a fixed point $x_{0}$, so that $f\left(x_{0}\right)=g\left(x_{0}\right)$. In fact, it is not even necessary that $f$ and $g$ commute.

The conjecture is also true if $f$, for example, is $1-1$ and $g(S) \subset f(S)$. For then $f^{-1} g$ takes $S$ into itself, and since $S$ is compact $f^{-1}$ is continuous, and hence $f^{-1} g$ is continuous. Thus $f^{-1} g$ has a fixed point $x_{0}$, and so $g\left(x_{0}\right)=f\left(x_{0}\right)$.

Finally, the conjecture is true if $S$ is one-dimensional, as we now prove.

THEOREM 21. Let I be the unit interval and $f, g: I \rightarrow I$ be continuous, commuting maps. Then there exists $x_{0} \in I$ such that $f\left(x_{0}\right)=g\left(x_{0}\right)$.

Proof. First note that if $f$ (say) is onto then the theorem is true. For there exist $a, b \in I$ such that $f(a)=0, f(b)=1$. Thus $(f-g)(a) \leqq 0$ and $(f-g)(b) \geqq 0$, implying $f=g$ at some $x$ between $a$ and $b$.

Now let $f$ and $g$ be any continuous commuting functions. Let $I_{n}=f^{n}(I)$. Then each $I_{n}$ is a compact interval and $I_{n+1} \subset I_{n}$. Let $I_{0}=\bigcap_{n=1}^{\infty} I_{n} \neq \varnothing$. Then $I_{0}$ is also a compact interval and we have, for $x \in I_{0}, x \in I_{n}$ and hence there exists $y_{n} \in I$ such that $x=f^{n}\left(y_{n}\right)$. Thus

$$
g(x)=g f^{n}\left(y_{n}\right)=f^{n}\left(g\left(y_{n}\right)\right) \in I_{n}
$$

and

$$
f(x)=f^{n+1}\left(y_{n}\right) \in I_{n+1} .
$$

It is clear then that $f$ and $g$ take $I_{0}$ into itself. If we can show that $f$ is onto on $I_{0}$, then the proof will be complete.

Let $x \in I_{0}$. Then $x \in I_{n}$ so that, as before, there is $y_{n} \in I$ such that $x=f^{n}\left(y_{n}\right)$. Thus

$$
x=f\left(f^{n-1}\left(y_{n}\right)\right)=f\left(z_{n}\right)
$$

where $z_{n}=f^{n-1}\left(y_{n}\right) \in I_{n-1}$. Let $z_{0}$ be a limit point of the sequence $\left\{z_{n}\right\}$. Then it is clear that $z_{0} \in I_{0}$, and since $f\left(z_{n}\right)=x$ for all $z_{n}$ we have $f\left(z_{0}\right)=x_{0}$. Thus $x \in f\left(I_{0}\right)$, completing the proof.

We now give an example to show the limitations on some of the theorems proved in the paper. Let $T_{\theta}$ be defined on the plane, using polar coordinates, by $T_{\theta}(\rho, \varphi)$ $=(\rho, \varphi+\theta)$. Then $\left\{T_{\theta}\right\}$ is a flow on any disk $S_{2}$ about the origin. If $S$ is any compact, convex set not containing 0 then $T_{2 \pi}(S)=S$. However, $T_{\theta}$ has no fixed point in $S$ for $\theta \neq 2 m \pi$. This shows that Theorem 18 cannot be extended to the case where $C_{0}=C_{1}$. 
Let $f_{1}=T_{2 \pi / m}$ in the above. Then for any compact, convex set $S$ not containing 0 we have $f_{1}^{m}(S) \subset S$ and, if $S$ is small enough, $f_{1}(S) \cap S=\varnothing$. It is clear that we can modify $f_{1}$ to a map $f$ such that $f^{m}\left(N_{\varepsilon}(S)\right) \subset S$ for some $\varepsilon>0$. But $f^{j}$ has no fixed point in $S$ for $j<m$. Thus it is seen that the number $c=g c f(N)$ in Theorem 12 is the best possible.

\section{REFERENCES}

1. F. E. Browder, On a generalization of the Schauder fixed point theorem, Duke Math. J. 26 (1959), 291-303. MR 21 \#4368.

2. - Another generalization of the Schauder fixed point theorm, Duke Math. J. 32 (1965), 399-406.

3. - A further generalization of the Schauder fixed point theorem, Duke Math. J. 32 (1965), 575-578.

4. J. Cronin, Fixed points and topological degree in nonlinear analysis, Math. Surveys, no. 11, Amer. Math. Soc., Providence, R. I., 1964. MR 29 \#1400.

5. J. Dugundji, An extension of Tietze's theorem, Pacific J. Math. 1 (1951), 353-367. MR 13, 373.

6. A. Granas, The theory of compact vector fields and some of its applications to topology of functional spaces. I, Rozprawy Mat. 30 (1962). MR 26 \#6743.

7. A. Halanaĭ, Asymptotic stability and small perturbations of periodic systems of differential equations with retarded arguments, Uspehi Mat. Nauk 17 (1962), no. 1 (103), 231-233. (Russian) MR 25 \#301.

8. W. A. Horn, A generalization of Browder's fixed point theorem, Abstract \#611-30, Notices Amer. Math. Soc. 11 (1964), 325.

9. G. S. Jones, The existence of periodic solutions of $f^{\prime}(x)=-\alpha f(x-1)\{1+f(x)\}$, J. Math. Anal. Appl. 5 (1962), 435-450. MR 25 \#5234.

10. — Asymptotic fixed point theorems and periodic systems of functional-differential equations, Contributions to Differential Equations 2 (1963), 385-405. MR 28 \#1361.

11. - Periodic motions in Banach space and applications to functional-differential equations, Contributions to Differential Equations 3 (1964), 75-106. MR 29 \#342.

12. - Hereditary dependence in the theory of differential equations. I, University of Maryland Tech. Note BN-385, 1965.

13. J. Schauder, Der Fixpunktsatz in Funktionalräumen, Studia Math. 2 (1930), 171-180.

14. A. Tychonoff, Ein Fixpunktsatz, Math. Ann. 111 (1935), 767-776.

\section{National Bureau of Standards,}

WASHINGTON, D. C. 20234 